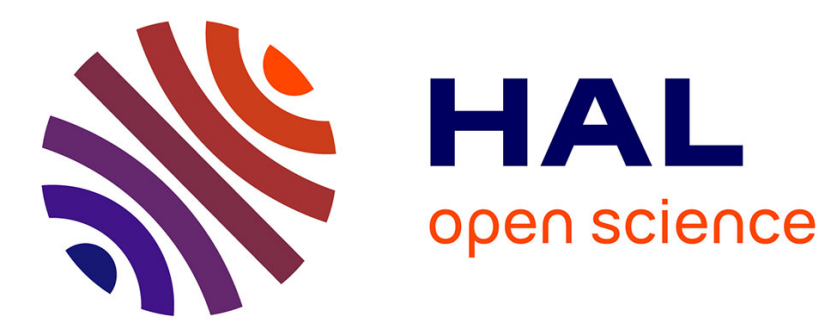

\title{
The spin glass to ferromagnetic order transition in AuFe alloys
}

\author{
A. Hamzić, I.A. Campbell
}

\section{To cite this version:}

A. Hamzić, I.A. Campbell. The spin glass to ferromagnetic order transition in AuFe alloys. Journal de Physique Lettres, 1981, 42 (13), pp.309-312. 10.1051/jphyslet:019810042013030900 . jpa-00231934

\section{HAL Id: jpa-00231934 https://hal.science/jpa-00231934}

Submitted on 1 Jan 1981

HAL is a multi-disciplinary open access archive for the deposit and dissemination of scientific research documents, whether they are published or not. The documents may come from teaching and research institutions in France or abroad, or from public or private research centers.
L'archive ouverte pluridisciplinaire HAL, est destinée au dépôt et à la diffusion de documents scientifiques de niveau recherche, publiés ou non, émanant des établissements d'enseignement et de recherche français ou étrangers, des laboratoires publics ou privés. 


\title{
The spin glass to ferromagnetic order transition in AuFe alloys
}

\author{
A. Hamzić and I. A. Campbell \\ Laboratoire de Physique des Solides, Université Paris-Sud, Bât. 510, 91405 Orsay, France \\ (Reçu le 3 avril 1981, accepté le 7 mai 1981)
}

\begin{abstract}
Résumé. - A partir de mesures de résistivité et de magnétorésistance sur des alliages AuFe, nous trouvons qu'audelà de la concentration où l'ordre ferromagnétique se manifeste, les ordres verre $\overline{\text { de }}$ spin et ferromagnétique coexistent à basses températures. Quand on augmente la température, on observe un minimum dans la susceptibilité au-dessus de la saturation technique. Ceci peut indiquer une transition vers un ordre ferromagnétique simple.
\end{abstract}

\begin{abstract}
From resistivity and magnetoresistance data on AuFe we conclude that above the concentration where ferromagnetic order appears, in the low temperature state ferromagnetic and spin glass like ordering coexist. As the temperature is increased a transition to normal ferromagnetic order is indicated by a well defined minimum in the susceptibility above technical saturation.
\end{abstract}

A number of magnetic alloy systems at low temperatures are spin glasses over a certain range of concentration but acquire some ferromagnetic order when the concentration of the magnetic component is increased sufficiently. The AuFe system is probably the best known example ; a number of different techniques have been used to study these alloys and a magnetic phase diagram has been given [1-3]. Below $C_{\text {crit }} \simeq 15 \% \mathrm{Fe}$ the alloys behave as spin glasses with the well known cusp in the initial susceptibility while above this concentration the magnetization behaviour resembles that of a normal ferromagnet. The regions on each side of the critical concentration have attracted attention; in the spin glass concentration range superparamagnetic effects have been observed above the cusp temperature $T_{\mathrm{g}}$ [3], and for $c>C_{\text {crit }}$ it has been suggested that the alloys are ferromagnets from $T_{\mathrm{c}}$ down to a second ordering temperature below which the systems are "spin glasses" [2]. This view has been contested on the basis of other low field magnetization measurements [4]. In addition neutron scattering results show that for these alloys above $C_{\text {crit }}$, there is an anomalous increase in the quasielastic neutron cross section as the temperature is lowered [1].

Traditionally these alloys have been described in terms of " magnetic clusters ». This terminology does not seem very appropriate in systems like these, dominated by long range competing interactions.

Here, we wish to show that resistivity and magnetoresistance measurements can help clarify the situation. Our data indicate that for $c>C_{\text {crit }}$, at low tem- perature and in zero applied field each local moment is randomly canted to some extent with reference to the overall magnetization direction, so ferromagnetic and spin glass order coexist. Then as the temperature is increased there is a well marked minimum in the susceptibility of the system above technical saturation implying a transition to a normal ferromagnetic state. This transition temperature is not the temperature at which the initial low field susceptibility reaches a plateau [2-4].

We can discuss the resistivity of magnetic alloys (such as $\mathrm{AuFe}$ ) using the model of Yoshida [5]. If the interaction between the magnetic atoms and the conduction electrons is $V-2 J \mathbf{S} . \mathbf{s}$ then the high temperature paramagnetic impurity resistivity is

$$
\rho_{\mathrm{HT}}=k c\left(V^{2}+J^{2} S(S+1)\right)
$$

where $k$ is a constant and $c$ is the concentration. At low temperatures an inelastic term $k c J^{2} S$ is frozen out $[5,6]$ and if there is a reduction in resistivity due to a ferromagnetic alignment arising either from interactions or from an applied field, then the resistivity becomes

$$
\rho=k c\left(V^{2}+J^{2} S^{2}-\frac{4 J^{2} V^{2} S^{2}}{V^{2}+J^{2} S^{2}}\left(\langle m\rangle_{\lambda} / S\right)^{2}\right) .
$$

Here $\langle m\rangle_{\lambda}$ is the ferromagnetic magnetization per atom averaged over the mean free path $\lambda$. We will write $\rho_{0}$ and $\langle m\rangle_{\lambda}^{0}$ respectively for the resistivity 
and average magnetization at zero temperature and in zero field. For a true spin glass $\langle m\rangle_{\lambda}^{0}=0$ so that last term in $\rho_{0}$ is zero, while for a true ferromagnet $\langle m\rangle_{\lambda}^{0}=S$.

The temperature dependence of the resistivity of AuFe alloys in zero field has been measured [7, 8]. In the concentration range up to about $8 \% \mathrm{Fe}$ the drop in impurity resistivity on cooling from $300 \mathrm{~K}$ to $0 \mathrm{~K}$ is small ; only the spin flip scattering is frozen out and the alloys at low temperature are true spin glasses with no ferromagnetic order in the scale of $\lambda$ [9]. As $C_{\text {crit }}$ is approached, $\rho_{\mathrm{HT}}-\rho_{0}$ increases dramatically and $\rho_{0}$ drops, figure 1 , showing that $\langle m\rangle_{\lambda}^{0} \neq 0$, i.e. short range ferromagnetic ordering on the scale of $\lambda$ or greater has begun to develop within the spin glass phase. From $8 \% \mathrm{Fe}$ to $C_{\text {crit }}$ is just the concentration range over which EPR results show superparamagnetic effects above the spin glass cusp temperature [3]. The superparamagnetic behaviour and the ferromagnetic short range ordering are clearly related.

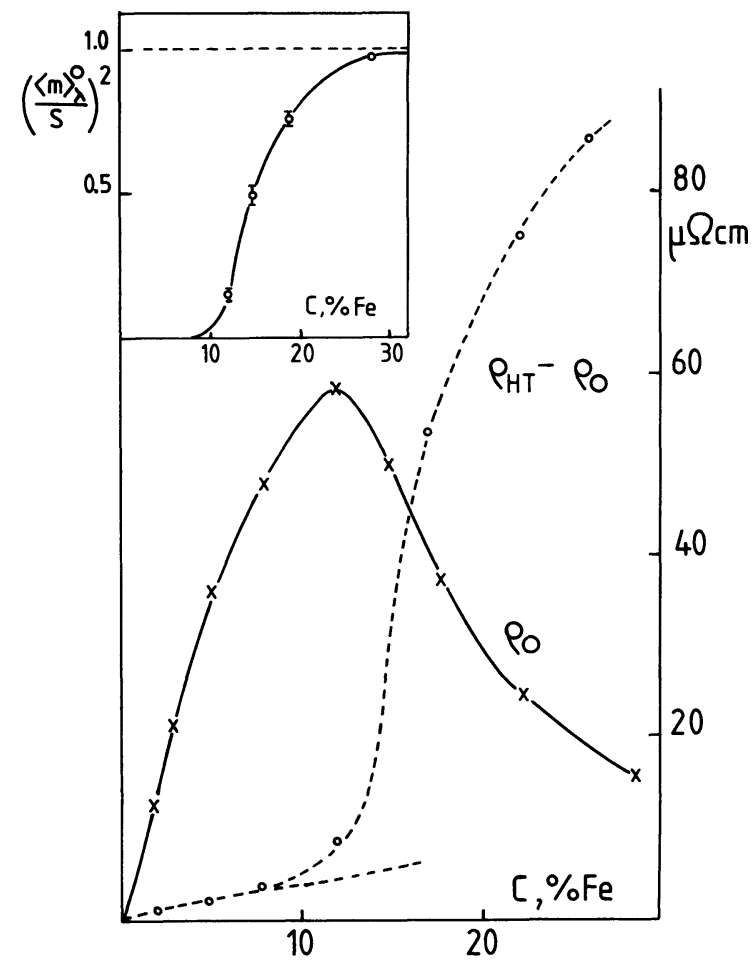

Fig. 1. - Residual resistivity $\rho_{0}$, and the difference between high temperature and residual resistivities $\rho_{\mathrm{HT}}-\rho_{0}$, for AuFe alloys as a function of Fe concentration (from Refs. [7] and [8]). Inset : local ferromagnetic order parameter $\left(\langle m\rangle_{\lambda}^{0} / S\right)^{2}$ (see text).

For $c>15 \%$ Fe these alloys have been referred to generally as "ferromagnets " and the high value of the resistivity change $\rho_{\mathrm{HT}}-\rho_{0}$ confirms a strong degree of ferromagnetic alignment. It is important to note that for all samples the zero field resistivity drops continuously as the temperature is lowered [7], so that the degree of ferromagnetic order is always increasing as the temperature drops. We can test if the ferromagnetic alignment is complete at low temperatures by measuring the magnetoresistance; if the spins are not fully ordered there will be a negative magnetoresistance as the magnetic field suppresses the residual disalignment. From equation (2)

$$
\left(\langle m\rangle_{\lambda}^{0} / S\right)^{2} \simeq 1+\Delta \rho_{\text {sat }} /\left(\rho_{\mathrm{HT}}-\rho_{0}-\Delta \rho_{\text {sat }}\right)
$$

where $\Delta \rho_{\mathrm{sat}}$ is the (negative) saturation magnetoresistance measured in a high magnetic field.

We have measured the magnetoresistance at $1.2 \mathrm{~K}$ up to $70 \mathrm{kG}$ on a number of AuFe samples. The samples were annealed at $800^{\circ} \mathrm{C}$ and quenched just before mounting. We observed strong negative isotropic magnetoresistance on all samples, together with weaker anisotropic magnetoresistance effects which we will not discuss here. The presence of the negative magnetoresistance shows that the spins are partially disaligned in zero field, even for $\mathrm{Au} 28 \% \mathrm{Fe}$. As the magnetoresistance is far from saturating in $70 \mathrm{kG}$ we can only give rough estimates of $\Delta \rho_{\text {sat }}$ and hence of $\left(\langle m\rangle_{\lambda}^{0} / S\right)^{2}$ through equation (3). These values are shown in the inset of figure 1 . We observe very much the same behaviour as for PtFe [10] where we suggested that the partially aligned state should be called a quasiferromagnet. We note, that the shape of the magnetoresistance curves at low temperature hardly varies with the $\mathrm{Fe}$ concentration - we need to apply as strong a field to fully saturate a $\mathrm{Au} 28 \% \mathrm{Fe}$ sample as a $\mathrm{Au} 15 \% \mathrm{Fe}$ sample although in zero field the former is very much closer to being fully magnetized than is the latter. This implies that the strength of the interactions acting against the ferromagnetic order are only weakly concentration dependent. Again the same behaviour was observed in PtFe [10] but for saturation fields twenty times smaller than in AuFe.

We have also measured the magnetoresistance of $\mathrm{Au} 19 \% \mathrm{Fe}$ as a function of the temperature from $\overline{1.2} \mathrm{~K}$ to $200 \mathrm{~K}$, figure 2 . As for a simple ferromagnet there is a sharp peak in $\mathrm{d} \rho / \mathrm{d} H$ at $T_{\mathrm{c}}$; however unlike normal ferromagnets the curve goes through a minimum and shows a second peak at $T=0$. The curve for $\mathrm{d} \rho / \mathrm{d} H$ bears a striking resemblance to the curve for the quasi-elastic neutron cross section of the same alloy [1] shown in the inset.

We can relate $\mathrm{d} \rho / \mathrm{d} H$ to the susceptibility through equation (2). Ignoring the field dependence of the spin flip scattering we have

$$
\mathrm{d} \rho / \mathrm{d} H \propto-\langle m(T)\rangle \chi(T) .
$$

This susceptibility $\chi(T)$ is the susceptibility per atom of the alloy above technical saturation; the initial susceptibility in weak fields which has been much studied in AuFe and other systems $[2-4,11]$ includes dominant domain effects which are certainly more complex than the intra-domain susceptibility we consider here. (The resistivity is not sensitive to domain rotations.) As we know the magne- 


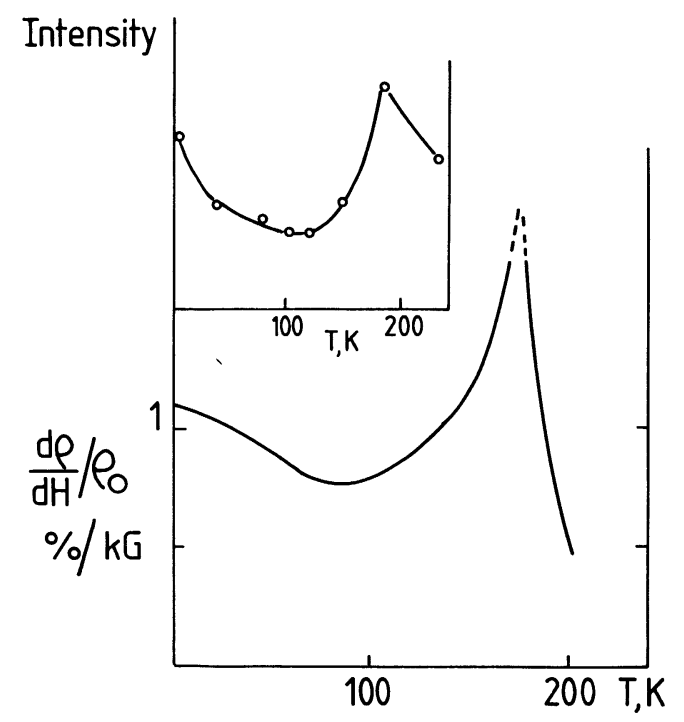

Fig. 2. - Magnetoresistance $\mathrm{d} \rho / \mathrm{d} H$ measured at $3 \mathrm{kG}$ applied fields for $\mathrm{AuFe} 19 \%$ as a function of temperature. Inset : quasielastic neutron scattering intensity, at $q=0.1 \AA^{-1}$, for $\mathrm{AuFe} 19 \%$, reference [1].

tization at technical saturation $\langle m(T)\rangle$ for this alloy [12] we can calculate $\chi(T)$, figure 3 . When the temperature is increased from zero, the susceptibility initially drops, before passing through a minimum and finally tending to infinity at $T_{\mathrm{c}}$ as it should for a ferromagnet.

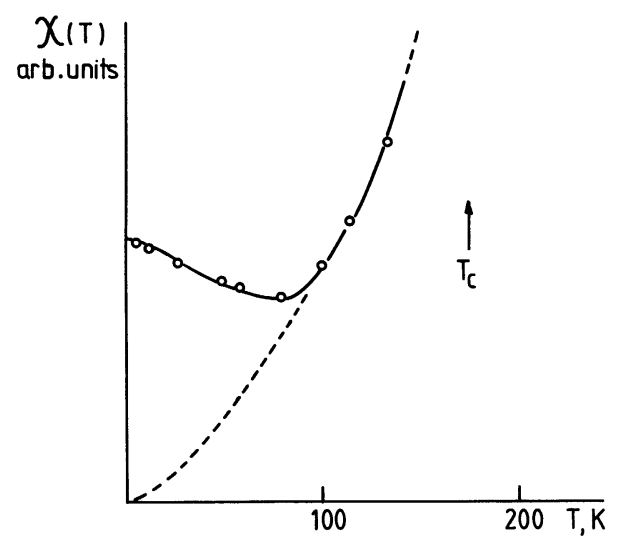

Fig. 3. - Susceptibility above technical saturation $\chi(T)$ as deduced from the magnetoresistance for AuFe $19 \%$ (see text). The dashed line indicates conventional ferromagnetic behaviour.

We can now review the information we have obtained on the alloys with $c>C_{\text {crit }}$. The first point is that the zero field resistivity $\rho(T)$ decreases smoothly and continuously as $T$ drops, so the degree of ferromagnetic order is always increasing as the temperature is lowered. No transition to a "spin glass" state is observed if the term "spin glass " implies a lower degree of ferromagnetic order. Secondly, perfect ferromagnetic order at zero temperature is never attained. Thirdly, there is a minimum in the temperature dependence of the susceptibility for $\mathrm{Au} 19 \% \mathrm{Fe}$ (and presumably the other alloys) related to an anomalous increase in the quasi-elastic neutron cross section at low temperatures [1]. In $\mathrm{CrFe}$ a similar quasi-elastic cross section curve was shown to be associated with a collapse of the conventional spin waves at low temperatures [13].

We suggest the following description : just below $T_{\mathrm{c}}$ the system behaves as a standard ferromagnet with for each spin $m_{z}^{i} \neq 0, m_{x}^{i}=m_{y}^{i}=0$. As the temperature drops the degree of ferromagnetic order increases continuously, but below some temperature the spins begin to acquire random uncorrelated canted moment components so $m_{x}^{i}, m_{y}^{i} \neq 0$ but $\left\langle m_{x}\right\rangle=\left\langle m_{y}\right\rangle=0$. This is essentially the "semi spin glass" state proposed by Villain [14]. Below this transition point, the conventional spin wave description starts to break down and the susceptibility of the alloy tends to increase; finally at low temperatures there is an overall average moment in the $z$ direction but local moments are randomly disaligned to some extent with respect to $z$ and the excitations are not conventional spin waves with well defined wave vectors.

The susceptibility curve of figure 3 would seem to indicate a sufficiently abrupt change of regime at $\sim 85 \mathrm{~K}$ to suggest a sharp transition temperature of this type. In a sense this ordering would correspond to a spin glass transition in the plane perpendicular to the average magnetization [14]. The perpendicular component will build up gradually as the temperature drops below the transition; the extra low temperature susceptibility, figure 3, may be thought of as due to the tilting of the perpendicular moment components by the magnetic field towards the $z$ direction, analogous to the perpendicular susceptibility of an antiferromagnet. Also, it is physically reasonable that spin waves no longer have well defined wave vectors in this low temperature phase.

We can compare the present susceptibility curve (susceptibility without the domain rotation effects) with the low field initial susceptibility data for alloys with a similar concentration [2-4]; these results showed a drop in the low field susceptibility at a lower temperature than $85 \mathrm{~K}(55 \mathrm{~K}[2]$ or $20 \mathrm{~K}[3,4])$ and we feel that this drop is not directly related to the transition we discuss but to some more complicated domain effect, which is probably also due to the competing nature of the interactions.

Theoretical phase diagrams have been given for the Sherrington-Kirkpatrick model $[15,16]$ in which a " replica symmetry breaking " transition occurs below a ferromagnetic ordering temperature. The projection hypothesis made by Parisi and Toulouse [16, 17] is that below the lower transition temperature the susceptibility becomes temperature independent. For a Heisenberg version of the Sherrington-Kirkpatrick model, there are two transitions below $T_{\mathrm{c}}$ [18] - a canting transition and then a transition of the same type as in the Ising model. Tentatively we can identify 
the model canting transition with the observed $85 \mathrm{~K}$ behaviour and the $20 \mathrm{~K}$ initial susceptibility drop with the lower model transition.

To summarize, we have used resistivity and magnetoresistance data to help clarify the complicated situation associated with the passage from spin glass to ferromagnetic ordering. The most novel point concerns the alloys above the critical concentration for « ferromagnetism "; we have evidence in favour of the existence of or «canting " transition well below the ferromagnetic ordering temperature, but distinct from a further lower transition characterized by a drop off in the low field initial susceptibility [2-4].

Acknowledgments. - We would like to thank Dr. W. Saslow, Dr. S. Senoussi and Dr. G. Toulouse for very useful discussions and Dr. E. Babic for providing the samples used.

\section{References}

[1] Murani, A. P., Roth, S., Radhakrishna, P., Rainford, B. D., Coles, B. R., Ibel, K., Goeltz, G. and Mezel, F., J. Phys. F 6 (1976) 425.

[2] Veerbeck, B. H. and Mydosh, J. A., J. Phys. F 8 (1978) L-111.

[3] Coles, B. R., Sarkissian, B. V. B. and TaYlor, R. H., Philos. Mag. B 37 (1978) 489.

[4] BECK, P., Solid State Commun. 34583.

[5] Yoshida, K., Phys. Rev. 107 (1957) 396.

[6] Fischer, K. M., Z. Phys. B 34 (1979) 45.

[7] Mydosh, J. A., Ford, P. J., KaWATRA, M. P. and Whall, T. E., Phys. Rev. B 14 (1976) 2057.

[8] Sundahl, R. C., Chen, T., Sivertsen, J. M. and Sato, Y., J. Appl. Phys. 37 (1966) 1024

[9] Campeell, I. A., Senoussi, S., Ousset, J. and Rakoto, H., to be published.
[10] Hamzić, A. and Campbell, I. A., J. Physique Lett. 42 (1981) L-17.

[11] Maletta, H. and Felsch, W., Z. Phys. B 37 (1980) 55

[12] Crangle, J. and Scott, W. R., J. Appl. Phys. 36 (1965) 921.

[13] Fincher, C. R., Shapiro, S. M., Palumbo, A. H. and Par Ks, R. D., Phys. Rev. Lett. 45 (1980) 474.

[14] Villain, J., Z. Phys. B 33 (1979) 31.

[15] De Almeida, J. R. L. and Thouless, D. J., J. Phys. A 11 (1978) 983.

[16] Toulouse, G., J. Physique Lett. 41 (1980) L-447.

[17] Parisi, G. and Toulouse, G., J. Physique Lett. 41 (1980) L-361.

[18] Gabay, M. and Toulouse, G., to be published. 